\title{
Human food chain and microorganisms: a case of co-evolution
}

\author{
M. Elisabetta Guerzoni* \\ Alma Mater Studiorum University of Bologna, Bologna, Italy \\ *Correspondence: elisabetta.guerzoni@unibo.it
}

The human food chain, from the raw materials to the human gut microbiota, can be regarded as a sequence of intercommunicating macro- and micro-ecosystems inhabited by more or less specialized microbial populations or consortia. The dynamics of development or selection of these different populations or individual species, including pathogens, spanned along the entire chain, as well as the effects of their metabolic activity on the food quality and safety, are matters of fundamental interest which involve also social and regulatory aspects. The borderlines of the well-known "reservoirs" or ecological niches of certain microbial species or ecotypes are destined to be widened. In fact the virtual human food chain tends to expand in time and space due to the extension of the products shelf life, the market globalization, the multiplicity of the geographic origin of the raw materials, which vehiculate pathogens and favor the spread of antibiotic resistant organisms. The diffusion of new thermal and non-thermal technological processes or new ingredients and the evolution of the distribution and consumption patterns require a continuous revision of the microbiological risk assessment. In fact, pathogen and spoilage organisms respond dynamically to their environment and its changes. Understanding their behavior is critical because of the evidence of increasing resistance to established sanitization and preservation techniques, and the increased use of minimal processes conditions, which are more vulnerable to the development of resistance. The evaluation of the risk in food safety requires prevision of the probability that microbial population sizes will not exceed defined levels at the consumption level (Food Safety Objectives). Predictive microbiology involves knowledge of the responses, in terms of survival, inactivation and growth, to environmental factors summarized as equations or mathematical models. Coupled with information on environmental history during processing and storage, predictive microbiology provides information in making decisions on the microbiological safety and quality of foods. However, food systems are colonized by microbial communities, which comprise an interwoven matrix of biological diversity undergoing physical or chemical variations over space and time. Relatively little is known about their structure and function and predictive modeling is limited by a dearth of comprehensive ecological principles that describe microbial community processes. During the last century the emphasis on the study of microbes in pure culture has isolated microorganisms from their communities and focused on their individual behavior in the biologically simple microenvironment of the experiment. Although these simple approaches have driven an explosion of knowledge in cellular processes and host-microbe interactions over the last two decades, now we have to focus the reality of food natural communities and complex assemblages as well (Little et al., 2008). It is necessary to explore the internal processes of microbial communities in an effort to begin to define the principles that underpin their ecological and evolutionary patterns. Defining these principles is necessary to enable predictive modeling of the dynamics of microbial communities in foods.

In contrast to their role as "enemies" the microorganisms can also act as "friends". The extraordinary precognition of the transcendentalist American poet Emerson that "more servants wait on man than he'll take notice of" evokes the incessant beneficial activity of the fermenting yeasts and lactic acid bacteria.

Food fermentation is the oldest form of food preservation and fermented foods have been produced and consumed for thousands of years and are strongly linked to human culture and traditions. It is estimated that fermented foods contributed to about one-third of the diet of worldwide and fermentation is a globally applied force for the preservation of a wide range of raw materials (cereals, roots, tubers, fruit and vegetable, milk, meat, fish etc.). Over the last few decades the field of fermented food has experienced a very fast development thanks to the applications of methods allowing precise picturing of their microbial ecology. In fact much research effort has been dedicated to the development and optimization of molecular culture-independent techniques, such as $16 \mathrm{~S}$ ribosomal DNA (rDNA) PCR, denaturating gradient gel electrophoresis (PCR-DGGE) or fluorescence in situ hybridization (FISH), for the reliable detection and monitoring of the microorganisms involved in the transformations activities (Giraffa and Carminati, 2008). Moreover the worldwide produced fermented products are regarded as valuable mines of microbial diversity and genes of biotechnological interest. Because of their economical potential, many of the microorganisms isolated and characterized during this last century have received considerable attention from the pharmaceutical and biotechnological industries. In particular the lactic acid bacteria and probiotic species play an essential role in the development of the dairy industry not only for the increasing diffusion of cheese production whose diversification numbers more than 700 kinds, which are manufactured worldwide, but also the tremendous increase of the human consumption of acidified/fermented milks. However, at present only a small number of suppliers throughout the world provide the starter market with a few strains whose general employment tends to flatten the taste and the flavor of the products.

Control of activity and functionality of microbial starters, as well as probiotic cultures under industrial fermentation conditions, is a crucial aspect for obtaining desirable and safe products. Acid, oxidative, chemical, osmotic stresses are just some of the harsh conditions that starter and nonstarter microorganisms have to manage to endure during fermentation processes. In order to respond to stressful situations all organisms have developed adaptative 
networks including stress responses. The changes in transcription rates, translation products and/or metabolism are some of the mechanisms in the "répertoire" of how the cells react to the stimuli. Understanding such mechanisms has become interesting for the food industry because of the need for defined and stable starter cultures. Major advances in high throughput technologies for the detection and quantification of nucleic acids, proteins, and metabolites have led to a paradigm shift in microbiological research. The new field of system biology attempts to integrate the complex data sets generated by high throughput approaches to develop a holistic understanding of complex biological structures and their responsiveness to external stimuli. System biology organizes data obtained by genomic, transcriptomic, proteomic, and metabolomic approaches to attempt to build descriptive and mechanistic models of integrative biological phenomena such as development or interactions of organisms with their environment (Kultz et al., 2007).

The microbial ability to respond to chemico-physical stresses is a pre-requisite also to survive in the human gut microbiota. Gibson (2002) defined the human gut microbiota "the end of the food chain and the start of good health". In fact its composition, through dependant on the host phenotype and history, is affected by the diet, prebiotic and probiotic intake, etc. The increasing awareness of the key role of this last link on human health and immune system has fueled an intense research activity on the effects of the flow of energy, nutrients and microorganisms, through the system, on the changes of the microbiota composition and its metabolism. Analytical approaches for understanding the compositions and functions of the intestinal microbiota are being developed to meet the challenges of the many tasks ahead (Nicholson et al., 2002; Ndagijimana et al., 2009).

Due to the different and contrasting roles of microorganisms in food quality, shelf life and safety, the research mission in Food microbiology should not be too narrowly defined.

Despite the diversity and complexity of this discipline, knowledge, prediction and control of the response mechanisms, growth and metabolic performances along the food chain and during the biotechnological processes can be seen as common aims and research priorities for food microbiologists. The achievement of such tasks requires also a multidisciplinary knowledge of the inter- and intra-specific communication mechanisms and of the interactions between microbial cells and food systems, their complexity, geometry, micro and macrostructure as well as chemico-physical variables and their changes over time.

\section{REFERENCES}

Gibson, G. (2002). Human gut microbiology: the end of the food chain or the start of good health? Microbiol. Today 29, 4-6.

Giraffa, G., and Carminati, D. (2008). "Molecular techniques in food fermentation: principle and applications," in Molecular Techniques in the Microbial Ecology of Fermented Foods, eds L. Cocolin and D. Ercolini (New York: Springer), 1-30.

Kultz, D., Fiol, D., Valkova, N., Gomez-Jimenez, S., Chan, S. Y., and Lee, J. (2007). Functional genomics and proteomics of the cellular osmotic stress response in "nonmodel” organisms. J. Exp. Biol. 210, 1593-1601.

Little, E. F., Robinson, C. J., Peterson, S. B., Raffa, K. F., and Handelsman, J. (2008). Rules of engagement: interspecies interactions that regulate microbial communities. Annu. Rev. Microbiol. 62, 375-401.

Ndagijimana, M., Laghi, L., Vitali, B., Placucci, G., Brigidi, P., and Guerzoni M. E. (2009). Effect of a synbiotic food consumption on human gut metabolic profiles evaluated by ${ }^{1} \mathrm{H}$ nuclear magnetic resonance spectroscopy. Int. J. Food Microbiol., 134, 147-153.

Nicholson, J. K., Connelly, J., Lindon, J. C., and Holmes, E. (2002). Metabonomics: a platform for studying drug toxicity and gene function. Nat. Rev. Drug Discov. 1, 153-161.

Received: 06 August 2010; accepted: 06 August 2010; published online: 06 October 2010.

Citation: Guerzoni ME (2010) Human food chain and microorganisms: a case of co-evolution. Front. Microbio. 1:106. doi:10.3389/fmicb.2010.00106

This article was submitted to Frontiers in Food Microbiology, a specialty of Frontiers in Microbiology.

Copyright (c) 2010 Guerzoni. This is an open-access article subject to an exclusive license agreement between the authors and the Frontiers Research Foundation, which permits unrestricted use, distribution, and reproduction in any medium, provided the original authors and source are credited. 